\title{
Postural responses to changing task conditions in patients with cerebellar lesions
}

\author{
P Mummel, D Timmann, U W H Krause, D Boering, A F Thilmann, H C Diener, \\ F B Horak
}

Department of Neurology, University of Essen, Germany

P Mummel

D Timmann

U W H Krause

H C Diener

Department of Neurology, Rhein/Ruhr Clinic, Auf der

Roetsch 2, Essen,

Germany

D Boering

A F Thilmann

Posture Control

Laboratory, RSD

Neurological Sciences

Institute, Portland,

OR, USA

F B Horak

Correspondence to: Dr Dagmar Timmann, Department of Neurology, University of Essen, Hufelandstrasse 55, 45122 Essen, Germany. Telephon 0049201723 2594; fax 0049201723 5901; email Dagmar.Timmann@uniessen.de

Received 23 December 1997 and in revised form 15 April 1998

Accepted 29 May 1998

\begin{abstract}
Objective-To investigate the role of the cerebellum in postural adaptation for changes to the stimulus type of support surface displacements (backward translations $v$ "toes up" rotations).

Methods-A group of 13 patients with chronic, isolated lesions of the cerebellum and 15 control subjects were tested. Automatic postural responses of the medial gastrocnemius and anterior tibial muscles were recorded. The first paradigm consisted of 10 rotational perturbations followed by 10 backward translations of the platform, and 10 backward translations followed by 10 rotations. The second paradigm consisted of 18 rotations and two randomly interposed translational perturbations, and 18 translations with two rotations randomly interposed.

Results-When the type of perturbation changed from an expected translation to an unexpected rotation and vice versa both control subjects and cerebellar patients showed an immediate and significant change in the response amplitude of the medial gastrocnemius and at the same time an immediate and significant change in the response amplitude of the anterior tibial muscles. Neither controls nor cerebellar patients showed effects of prediction in surface displacements of unexpected types of perturbation. Both controls and cerebellar patients showed no gradual increase in the gastrocnemius response in subsequent trials of surface translations following a block of 10 surface rotations and no gradual increase in the response amplitude of the anterior tibial muscle in subsequent trials of surface rotations following a block of 10 surface translations.

Conclusions-Despite postural hypermetria, the integrity of the cerebellum does not seem critical for adaptation of postural synergies to changing stimulus types of surface displacements. The present results support previous findings suggesting that the main role of the cerebellum in automatic postural responses may be gain control.

(F Neurol Neurosurg Psychiatry 1998;65:734-742)
\end{abstract}

Keywords: posture; cerebellum; human; adaptation

Rapid automatic postural responses are evoked whenever there is an external perturbation to a body segment that causes disequilibrium or alters postural orientation. ${ }^{1}$ Exposing subjects who are standing on a movable platform to a backward horizontal surface perturbation endangering them to fall forward, for example, leads to a rapid and stereotyped response in the medial gastrocnemius muscle. To be functional, postural responses must adapt or change when biomechanical and sensory conditions change. A postural response in the medial gastrocnemius would be functionally inappropriate for stabilising sway when the type of perturbation changes from a backward translation to an upward rotation with the possibility of falling backwards, despite the fact that both perturbations result in ankle dorsiflexion.

Nashner reported that normal subjects progressively alter postural response magnitude in the course of the succeeding three to five trials after an unexpected change in the type of the surface displacement (translation $v$ rotation). ${ }^{2}$ He suggested that the "functionally inappropriate" gastrocnemius muscle response sequentially disappeared to upward rotations after backward translations, whereas the "functionally appropriate" gastrocnemius muscle response sequentially increased to backward translations after upward rotations.

A possible cerebellar involvement in adaptation of postural synergies has been suggested by Nashner. He proposed that the ability of cerebellar patients to adapt their postural responses after a change in the perturbation type was severely impaired. ${ }^{23}$ In addition, Nashner and Grimm reported inconsistent and delayed timing of postural muscle responses to surface displacements in patients with cerebellar and other central lesions. ${ }^{3}$

However, later experiments showed that normal subjects show an immediate change in the response amplitude at the first trial when the type of perturbation changes. ${ }^{4-8}$ Although Nashner's findings of progressive alterations of postural responses to changes in types of perturbations over succeeding trials have already been challenged in healthy subjects, Nashner and Grimm's findings of impaired adaptation of postural responses to changing perturbation types in cerebellar patients are often used to support the role of the cerebellum in adaptation of movement synergies. ${ }^{9}{ }^{10}$ Nashner and Grimm's study included patients with additional extracerebellar lesions. Their findings of increased latencies, for example, have been challenged in recent studies examining patients with circumscribed cerebellar lesions. ${ }^{11} 12$ 
Recent studies from our group suggest that the main cerebellar function in early automatic postural responses might be restricted to gain control. For example, early postural responses are known to be centrally modified by directionally specific central set effects using prediction based on prior amplitude experience. ${ }^{13}$ Timmann and Horak showed that the role of the cerebellum in set dependent amplitude scaling of postural responses relates to its importance in accurately modifying response gain based on prior experience. ${ }^{14}$

The aim of the present study was to further support the hypothesis that the role of the cerebellum in the control of automatic postural responses is restricted to gain control. We hypothesised that cerebellar patients' ability to change postural synergies to changing stimulus types was not affected despite their hypermetria. In the first part of our study the role of the cerebellum in the adaptation of postural synergies to rotational perturbations after platform translations and to translational perturbations after platform rotations was reinvestigated in a group of patients with lesions restricted to the cerebellum. In the second part of the study possible effects of prediction were investigated in expected and unexpected trials of platform rotations and translations in control subjects and cerebellar patients.

\section{Materials and methods}

PATIENTS

A total of 15 cerebellar patients were tested. Two patients were excluded from the final analysis because of additional signs of peripheral neuropathy. The remaining 13 patients, four women and nine men with a mean age of 56.4 (range 26-68) years presented with chronic, isolated lesions of the cerebellum (table 1). Two cerebellar patients had alcohol induced anterior lobe syndrome, five patients had a cerebellar infarction, and four had idiopathic late onset ataxia (IDCA; three with an age of onset over 50 years)..$^{15}$ Two patients had had a cerebellar tumour removed. All 13 patients showed mild to moderate ataxia of gait, anterior/posterior sway in stance with eyes closed, and mild to moderate dysmetria in the heel-to-shin manoeuvre based on a scale adapted from Klockgether et al. ${ }^{16}$ None of these patients had extracerebellar signs or significant

Table 1 Clinical data for cerebellar patients

\begin{tabular}{lllllll}
\hline Patie $n t$ & Age $(y)$ & Sex & Diagnosis & $\begin{array}{l}\text { Ataxia of } \\
\text { gait }^{\star}\end{array}$ & $\begin{array}{l}\text { Ataxia of } \\
\text { stance }\end{array}$ & $\begin{array}{l}\text { Lower limb } \\
\text { ataxia }^{\star}\end{array}$ \\
\hline 1 & 49 & M & Left cerebellar infarction & 2 & 1 & 2 \\
2 & 59 & M & Left cerebellar infarction & 1 & 1 & 1 \\
3 & 49 & M & Right cerebellar infarction & 1 & 1 & 0 \\
4 & 66 & M & Right cerebellar infarction & 2 & 2 & 1 \\
5 & 67 & F & Right cerebellar infarction & 3 & 3 & 2 \\
6 & 45 & F & Right cerebellar tumour & 4 & 3 & 3 \\
7 & 26 & M & Right cerebellar tumour & 1 & 0 & 0 \\
8 & 57 & M & Alcoholic cerebellar atrophy & 2 & 2 & 1 \\
9 & 65 & M & Alcoholic cerebellar atrophy & 2 & 2 & 3 \\
10 & 54 & M & IDCA $\dagger$ & 3 & 2 & 2 \\
11 & 64 & F & IDCA & 3 & 2 & 3 \\
12 & 65 & F & IDCA & 3 & 2 & 3 \\
13 & 68 & M & IDCA & 3 & 2 & 3 \\
& & & &
\end{tabular}

*Adapted from Klockgether et al [16]: $0=$ Absent; 5=most severe.

†IDCA=idiopathic cerebellar ataxia. orthopaedic or psychological constraints that could affect posture.

A group of 15 control subjects (three women, 12 men) with a comparable age range (mean age 48.8, range 24-70 years) and without neurological or orthopaedic limitations were tested. All subjects had a complete neurological evaluation by one of us. Informed consent from patients and normal subjects and approval of the local ethics committee were obtained.

METHODS

Subjects stood upright on a moveable forceplate $\left(\right.$ NeuroCom $\left.{ }^{\circledR}\right)$. They stood with arms folded in front across the waist, eyes open and feet $10-20 \mathrm{~cm}$ apart at the heels. The methods have previously been described in detail. ${ }^{17}{ }^{18}$ Safety straps were fitted loosely enough to prevent the patient from gaining support. Postural perturbations consisted of backward translations or dorsiflexion rotations ("toes up") of the platform. Dorsiflexion rotations were made colinear with the ankle axis at an amplitude of $4^{\circ}$ and a rate of $50 \%$ s. Backward-ramp translations of the platform were made with an amplitude of $2.7 \mathrm{~cm}$ and a constant velocity of 40 $\mathrm{cm} / \mathrm{s}$. Muscle activities of the medial gastrocnemius and tibialis anterior were recorded bilaterally using $2.5 \mathrm{~cm}$ surface electrodes spaced $2-4 \mathrm{~cm}$ apart. In healthy subjects and in patients with bilateral cerebellar lesions the results of the right leg were analysed. In patients with unilateral lesions, the results obtained in the leg ipsilateral to the side of cerebellar impairment (left $n=2$; right $n=5$ ) were used for statistical analysis. The EMG signals were amplified, full wave rectified, bandpass filtered $(10 \mathrm{~Hz}-10 \mathrm{kHz})$, and stored on hard disk for off line analysis (sampling rate $=1 \mathrm{kHz}$, resolution $=16$ bytes).

\section{PROTOCOL}

All subjects were exposed to the same order of two different protocols each consisting of two sequences of 20 trials (total of 80 trials). Subjects were instructed to maintain an upright posture. They were informed that they would receive perturbations of the platform. Subjects were not informed about the stimulus type.

The time between trials varied unpredictably between 15 and 30 seconds. Based on visual inspection of the subject's posture, the time interval was determined by the examiner after the subject returned to a quiet, stabilised position. The buttons of the keyboard were pressed carefully to prevent any possible auditory cues signalling to the subject the beginning of a perturbation or a change in perturbation type.

\section{Protocol 1: blocked}

The first test sequence consisted of 10 rotational perturbations followed by 10 backward translations of the platform. After a 5 minute rest, the second sequence consisted of 10 translations followed by 10 rotations.

Protocol 2: expected and unexpected

The third test sequence consisted of 18 rotations and two randomly interposed transla- 
tional perturbations (unexpected trials, trial number 7 and 17). In the fourth sequence, the subjects were exposed to a block of 18 backward translations in which two rotations (unexpected trials, trial number 5 and 13) were randomly inserted. In each block the trial preceding an unexpected trial was selected to represent the expected condition.

DATA ANALYSIS

The EMG data were collected for 1 second including $250 \mathrm{~ms}$ before the perturbation. The EMG latencies were identified by visual inspection and by placing the cursor at the earliest time that EMG activity deviated from the predisplacement EMG baseline level. Due to difficulties in defining the end point of postural responses, the integrated EMG (IEMG) was

Control group
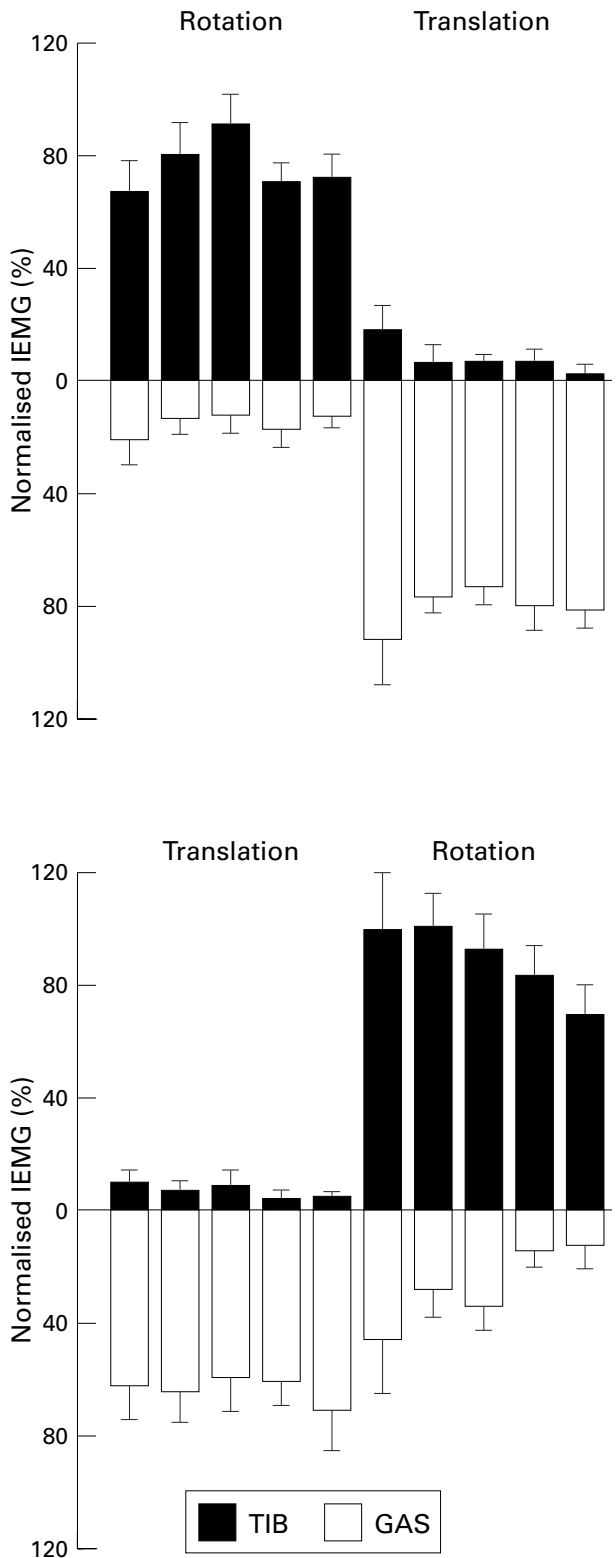

calculated using fixed intervals from the onset of the burst $(0-75 \mathrm{~ms})$. In protocol 2 ("expected and unexpected) a second fixed interval (0-25 ms) was introduced because no effects of prediction were demonstrated using a fixed interval of $75 \mathrm{~ms}$. The muscle activity for $100 \mathrm{~ms}$ before the onset of platform movement was used as an estimate of the tonic background activity of the muscle. The average amplitude of the tonic background estimate was subtracted from the response amplitude to insure an accurate measure of the response amplitude and to account for variations in the background activity during the experimental session. The IEMGs were normalised by assigning an arbitrary value of $100 \%$ to each subject's mean IEMG values for gastrocnemius (average of 10 translations) and tibialis anterior

Cerebellar group

Rotation

Translation

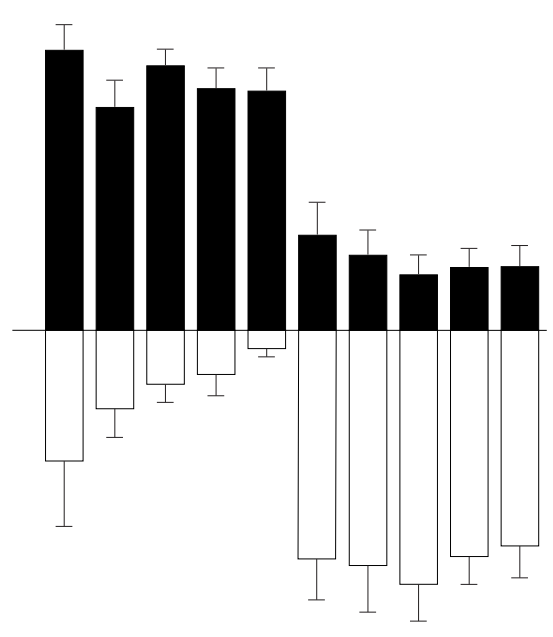

Translation

Rotation

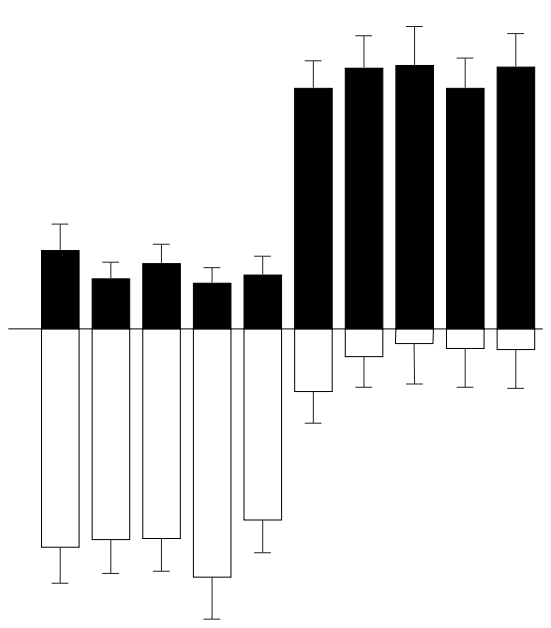

Figure 1 Group means (SD) of normalised tibial anterior IEMG (filled columns) and normalised medial gastrocnemius IEMG (open columns) for five sequential trials of surface translations (trials 11-15), following a block of five surface rotations (trials 6-10) (top), respectively five sequential trials of surface rotations (trials 11-15), following a block of five surface translations (trials 6-10) (bottom). 
A

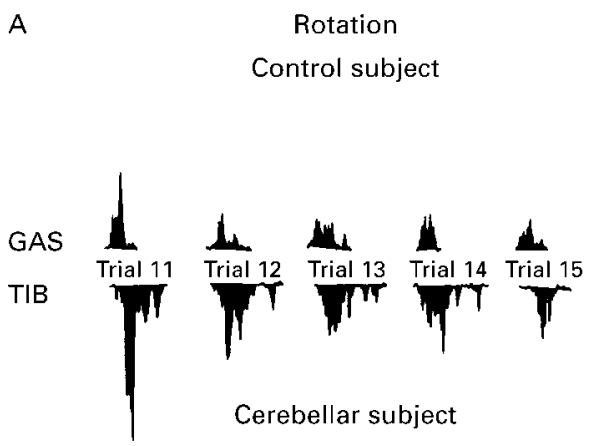

TIB

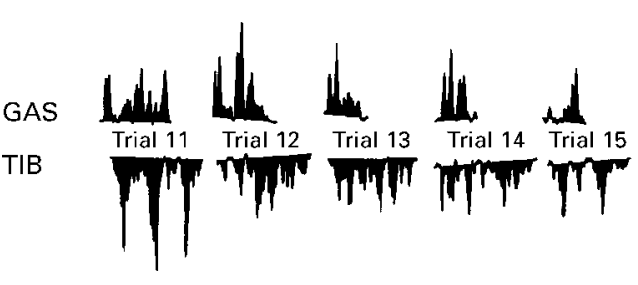

B

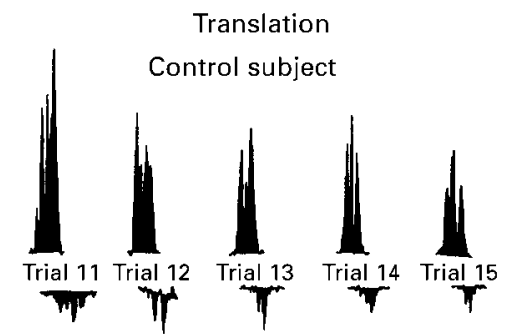

GAS

Figure 2 Gastrocnemius and tibialis anterior EMG activity for five subsequent trials (trials 11-15) of surface rotations following translations $(A)$ and of surface translations following rotations $(B)$ in a representative control (top) and cerebellar patient (bottom). Both the control and cerebellar patient showed a tendency to gradually reduce the magnitude of postural responses of the gastrocnemius muscle (and tibialis anterior) with repeated exposure to platform rotations following platform translations $(A)$. Furthermore, both the control and cerebellar patient showed a tendency to gradually reduce the magnitude of postural responses of the tibialis anterior (and gastrocnemius muscle) with repeated exposure to platform translations following platform rotations (B).

(average of 10 rotations) over a fixed time window (0-75 ms) in one condition (first sequence in protocol 1: 10 rotations followed by 10 translations) and by referencing the changes to these values. Only normalised EMG data were entered into statistical analysis to eliminate the effect of hypermetria in the group of cerebellar patients. To minimise the effects of "startlelike" responses, we did not analyse the first five trials of the serial presentations.

Protocol 1: blocked

From the first two sequences, each subject's gastrocnemius muscle and tibialis anterior IEMG values from trials 6-10 (sequence 1: rotations, sequence 2 : translations) were compared with their IEMG values from trials 11-15 (sequence 1: translations, sequence 2: rotations). The effects of stimulus type (translation $v$ rotation), sequence of perturbations (translation/rotation $v$ rotation/translation), group (control $v$ cerebellar), and trial were compared using a repeated measures factors design (MANOVA, SPSS statistical package). The repeated measures design was set up as follows: dependent variables=tibialis anterior and gastrocnemius IEMG; repeated measures factors=stimulus type, sequences, trial; between group factor=control, cerebellar group. Initially the assumption of constancy of variance of variables was tested using Bartlett's test of homogeneity. Greenhouse-Geisser's adjustment of degrees of freedom was applied to correct for small departures from the assumption of normality and equality of variance in the three factor design.

For post hoc comparisons of the first and second trial of translations following rotations (and of rotations following translations) paired $t$ tests were performed in both the control and cerebellar group. $p$ Values for effects were set at $<0.05$.

\section{Protocol 2: expected and unexpected}

Differences between responses to expected and unexpected conditions were calculated by comparing each subject's mean gastrocnemius IEMG value of trials 4 and 12 in the fourth sequence (expected translations) to the mean gastrocnemius muscle IEMG value of trials 7 and 17 from the third sequence (unexpected translations) and by comparing each subject's mean tibialis anterior IEMG value of trials 6 and 16 in the third block (expected rotations) to the mean tibialis anterior IEMG value of trials 5 and 13 from the fourth sequence (unexpected rotations).

Differences (" $\Delta$ values") were calculated between the mean gastrocnemius muscle values in expected and unexpected translational conditions and between the mean tibialis anterior values in expected and unexpected rotational conditions for each integration window (0-25 ms and 0-75 ms) and for each individual subject. All $\Delta$ values for each variable and each group were tested for being significantly different from 0 (two tailed, one sample $t$ test). Differences between groups were tested for significance with two tailed, unpaired $t$ tests. p Values for effects were set at $<0.0125$ (Bonferroni correction was used to account for multiple comparisons). Initially the assumption of constancy of variance of variables was tested using Bartlett's test of homogeneity.

\section{Results}

The gastrocnemius and tibialis anterior muscle response latencies were not significantly differ- 


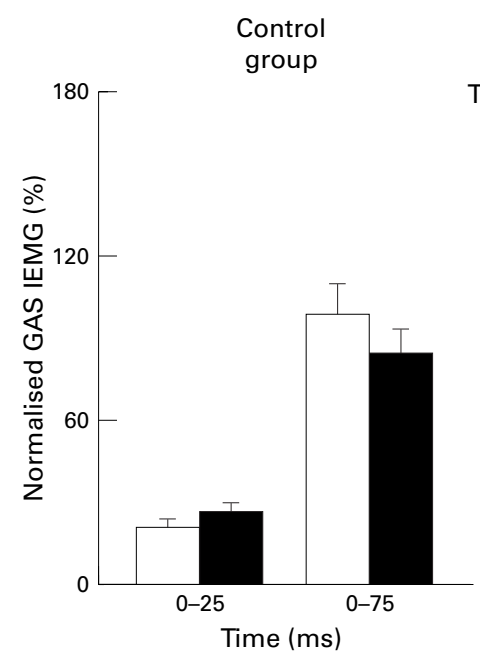

Translation

Cerebellar group
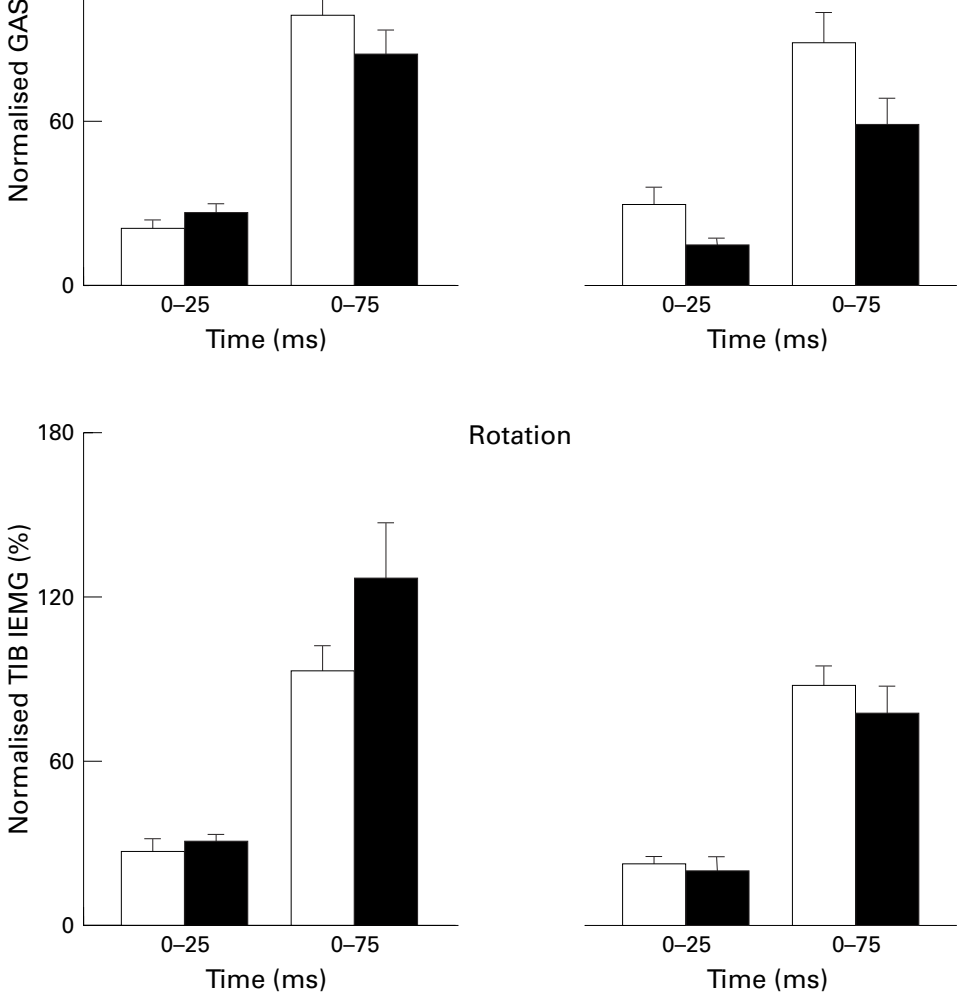

Rotation

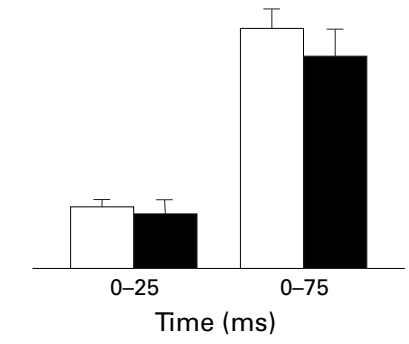

Expected Unexpected

Figure 3 Group means (SD) of normalized early (0-25 ms) and late (0-75 ms) gastrocnemius muscle IEMG for expected (open columns) and unexpected (filled columns) translational perturbations and of normalised early $(0-25 \mathrm{~ms})$ and late $(0-75 \mathrm{~ms})$ tibialis anterior IEMG for expected (open columns) and unexpected (filled columns) rotational perturbations. ent between the control and cerebellar groups $(\mathrm{p}>0.05)$.

The long latency response in the shortened tibialis anterior muscle in response to platform rotations occurred at 114 (SD 31) $\mathrm{ms}$ in control subjects. Medium latency response in the stretched gastrocnemius muscle showed a trend (non-significant) towards being earlier, at 97 (SD 37) ms. The latencies of muscle activation in response to platform translations were similar to the latencies for rotations. The mean response latencies to horizontal perturbations were 89 (SD 25) $\mathrm{ms}$ for the gastrocnemius muscle and 110 (SD 37) $\mathrm{ms}$ for the tibialis anterior muscle in the control group.

The cerebellar patients showed similar latencies of muscle activation in response to platform rotations and translations as the normal subjects. The long latency response in the shortened tibialis anterior muscle in response to rotations occurred at 108 (SD 37) $\mathrm{ms}$ and the medium latency responses in the stretched gastrocnemius muscle muscle occurred at 88 (SD 39) ms. The mean latency responses to the horizontal perturbations were 87 (SD 34) ms for the gastrocnemius and 114 (SD 38) $\mathrm{ms}$ for the tibialis anterior in the cerebellar group. Cerebellar patients had much larger postural response magnitudes than normal subjects (see figs 2 and 4). Similarly to normal subjects, cerebellar patients showed a large and consistent response in the gastrocnemius to a horizontal perturbation and a large and consistent response in the tibialis anterior to a rotational perturbation. Unlike control subjects, who seldom activated the tibialis anterior muscle in response to a horizontal perturbation (three subjects out of 15), cerebellar patients more often activated the ventral antagonist muscle in response to a translational movement (eight patients out of 13). Similarly to control subjects, cerebellar patients had much larger gastrocnemius muscle responses to translations than to rotations.

Protocol 1: blocked

Both cerebellar patients and control subjects showed an immediate and significant decrease in the response amplitude of the tibialis anterior muscle (mean IEMG decrease: cerebellar $65.8 \%$, control $59.4 \%$ ) and, at the same time, an immediate and significant increase in the response amplitude of the gastrocnemius muscle (mean IEMG increase: cerebellar $86.7 \%$, control $92.8 \%$ ) when the stimulus type was changed from rotations to translations. The mean averages of trials $6-10$ and $11-15$ for both the control and cerebellar group are shown in figure 1 (top). When the type of perturbation changed from translation to rotation, both cerebellar patients and normal subjects showed an immediate and significant decrease in the response amplitude of the gastrocnemius muscle (mean IEMG decrease: cerebellar $68.1 \%$, control $59.5 \%$ ) and at the same time an immediate and significant increase in the response amplitude of the tibialis anterior muscle (mean IEMG increase: cerebellar $83.3 \%$, control $104.1 \%$ ) (fig 1, bottom).

When the type of perturbation changed, all control subjects and cerebellar patients changed the postural response pattern within one or two trials. In the first rotational trial following platform translations, tibialis anterior IEMG activity increased and gastrocnemius muscle IEMG activity decreased, except in one cerebellar patient (No 10, table 2), in whom gastrocnemius muscle IEMG decreased within the second rotational trial. In the first translational trial after platform rotations, gastrocnemius muscle IEMG increased and tibialis anterior IEMG decreased within one trial in $13 / 15$ control and $11 / 13$ cerebellar patients. In two control subjects (Nos 3 and 11; table 2) and two cerebellar patients (Nos 10 and 13; table 2) tibialis anterior IEMG decreased within the second trial.

Figure 1 indicates that neither control subjects nor cerebellar patients showed a gradual increase in ("functionally appropriate") gastrocnemius muscle responses to translational following rotational movements, or in ("functionally appropriate") tibialis anterior responses to rotational following translational 
Table 2 Difference in the size of normalised gastrocnemius (GAS) and tibialis anterior (TIB) IEMG (\%) comparing the first trial of platform translation following platform rotations (left) and the first trial of rotation following translations (right) for all controls and cerebellar patients.

\begin{tabular}{|c|c|c|c|c|c|c|c|c|c|}
\hline \multicolumn{5}{|c|}{ Control subjects } & \multicolumn{5}{|c|}{ Cerebellar patients } \\
\hline \multicolumn{3}{|c|}{ Translation following rotations } & \multicolumn{2}{|c|}{$\begin{array}{l}\text { Rotation following } \\
\text { translations }\end{array}$} & \multicolumn{3}{|c|}{ Translation following rotations } & \multicolumn{2}{|c|}{$\begin{array}{l}\text { Rotation following } \\
\text { Translations }\end{array}$} \\
\hline No & GAS & TIB & GAS & TIB & No & GAS & TIB & GAS & TIB \\
\hline 1 & 240.6 & -87.9 & -39.7 & 92.7 & 1 & 133.7 & -94.8 & -85.2 & 124.3 \\
\hline 2 & 69.9 & -60.4 & -51.8 & 69.3 & 2 & 69.2 & -49.3 & -43.2 & 99.8 \\
\hline 3 & 86.6 & 4.2 & -10.3 & 55.0 & 3 & 60.4 & -96.7 & -134.7 & 79.4 \\
\hline 4 & 75.3 & -73.4 & -77.1 & 107.4 & 4 & 84.0 & -26.3 & -23.4 & 93.2 \\
\hline 5 & 172.3 & -39.5 & -112.2 & 271.9 & 5 & 3.54 & -6.6 & -111.7 & 76.7 \\
\hline 6 & 143.7 & -53.7 & -53.1 & 56.2 & 6 & 38.9 & -112.4 & -113.9 & 57.3 \\
\hline 7 & 142.3 & -34.8 & -65.6 & 48.5 & 7 & 127.3 & -74.2 & -5 & 123.7 \\
\hline 8 & 120.6 & -18.7 & -125.0 & 232.2 & 8 & - & -104.4 & - & 58.0 \\
\hline 9 & 65.4 & -124.6 & -64.7 & 89 & 9 & 53.1 & -84.0 & -24.6 & 43.2 \\
\hline 10 & 57.9 & -79.9 & - & 70.8 & 10 & 200.3 & 63.8 & 83.9 & 52.7 \\
\hline 11 & 116.7 & 6.8 & - & - & 11 & 65.3 & -45.9 & - & - \\
\hline 12 & 58.3 & -85.1 & -15.2 & 52.7 & 12 & 66.2 & -58.3 & -40 & 82.3 \\
\hline 13 & 88.2 & -65.7 & -46.6 & 206.2 & 13 & 139.3 & 32.9 & - & - \\
\hline 14 & 65.9 & -56.0 & -252.0 & 74.3 & & & & & \\
\hline 15 & 110.5 & -89.7 & - & - & & & & & \\
\hline
\end{tabular}

movements. Rather, both groups showed a tendency to gradually reduce the magnitude of the postural response of the gastrocnemius muscle and tibialis anterior with repeated exposure to the same surface perturbation.

Multivariate analysis of variance (MANOVA) disclosed a significant effect of stimulus type for both tibialis anterior and gastrocnemius muscle IEMG (tibialis anterior $F$ $(1,26)=179.7, \mathrm{p}<0.0001$; gastrocnemius muscle $F(1,26)=197.2, \mathrm{p}<0.001)$. There were no significant effects of group (tibialis anterior $F$ $(1,26)=0.03, \mathrm{p}=0.86$; gastrocnemius muscle: $F$ $(1,26)=1.19, \mathrm{p}=0.67)$ and sequence (tibialis anterior $F(1,26)=1.84, \mathrm{p}=0.18$; gastrocnemius muscle: $F(1,26)=2.24, \mathrm{p}=0.14)$ ). The trial effect was significant for tibialis anterior IEMG $\quad(F \quad(2.95,76.6)=2.96, \quad \mathrm{p}=0.038$; Greenhouse-Geisser adjustment for multiple degrees of freedom). The interaction of stimulus type and trial effect reached significance $(F$ $(3.36,87.3)=2.52, \mathrm{p}=0.057)$. A significant interaction of stimulus type and trial effects would suggest that the significant main effect of stimulus type was not the same at all levels of trial. However, neither the trial effect $(F(2.12$, $55.1)=1.83, p=0.13$ ) nor the interaction of stimulus type and trial effect for gastrocnemius muscle IEMG $(F(2.72,70.7)=1.32, \mathrm{p}=0.27)$ reached significance.

Representative gastrocnemius and tibialis anterior muscle EMG examples are shown in fig 2 . In both the control subject and cerebellar patient, the "functionally appropriate" tibialis anterior response did not sequentially increase to surface rotations following translations (fig 2 A) and the "functionally appropriate" gastrocnemius muscle response did not sequentially increase to surface translations following rotations (fig 2 B). The magnitude of the gastrocnemius muscle response showed a tendency to gradually reduce with repeated exposure to platform rotations following platform translations (fig $2 \mathrm{~A}$ ) and of the tibialis anterior response with repeated exposure to platform translations following platform rotations (fig 2 B).
An initial reduction between trial 1 and subsequent trials has been related to habituation of a startle-like response. ${ }^{4}$ Paired $t$ tests were calculated to test for possible significant reductions between the first and second trial following a change in stimulus type. The reduction in initial gastrocnemius muscle IEMG between the first and second trials of platform rotations following platform translations was significant in the cerebellar group only (cerebellar group: $\mathrm{p}=0.006$; control group: $\mathrm{p}=0.35$ ). There was no significant reduction in initial tibialis anterior IEMG between the first and consequent trials of platform translations following platform rotations (control group $\mathrm{p}=0.99$, cerebellar group $\mathrm{p}=0.48$ (paired $t$ test)).

Protocol 2: expected and unexpected

No significant differences were found between expected and unexpected gastrocnemius muscle and tibialis anterior response magnitudes to rotational platform movements that could point to the use of prediction based on prior experience. Group data are shown in fig 3 (bottom) and representative examples in fig 4 . $\Delta$ Values of integrated EMGs for expected and unexpected rotational conditions were not significantly different from zero for both the control (0-25 ms: $p=0.66,0-75 \mathrm{~ms}: \mathrm{p}=0.09)$ and cerebellar group $(0-25 \mathrm{~ms}: \mathrm{p}=0.74,0-75 \mathrm{~ms}$ : $\mathrm{p}=0.24)$. There were also no significant differences in $\Delta$ values between groups $(0-25 \mathrm{~ms}$ : $\mathrm{p}=0.57,0-75 \mathrm{~ms}: \mathrm{p}=0.05$; $\mathrm{p}$ values for effects were set at $<0.0125$ (Bonferroni correction applied to account for multiple comparisons)). However, there was a tendency of the ("functionally appropriate") tibialis anterior response to be larger in unexpected compared with expected rotations in control subjects suggesting response "habituation" with repetition (fig 3, bottom).

Similarly, both cerebellar patients and control subjects did not show any effect of prediction based on prior experience, comparing expected and unexpected translational perturbations (fig 4 (top)). $\Delta$ Values of integrated EMGs for expected and unexpected transla- 
Control subject

Translation

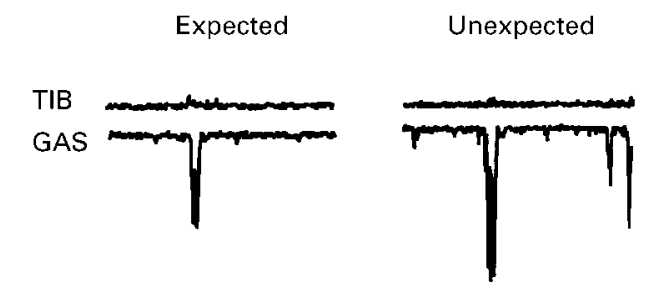

Rotation

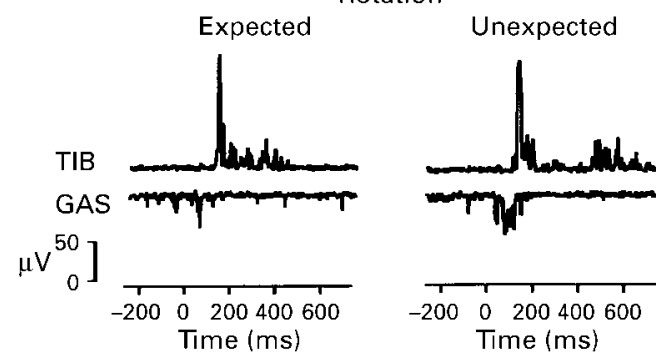

Cerebellar subject

Translation

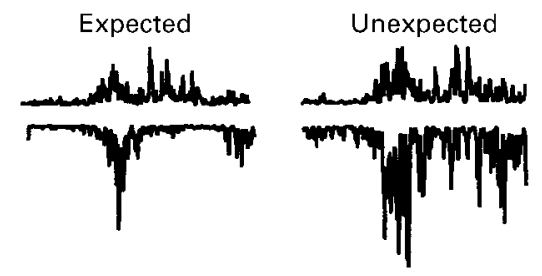

Rotation

Expected Unexpected

Figure 4 Tibialis anterior and gastrocnemius muscle EMG activity after an expected and unexpected translational perturbation (top) and expected and unexpected rotational perturbation (bottom) in a representative control (left) and cerebellar patient (right). There was no significant reduction of the gastrocnemius muscle EMG in unexpected compared with expected platform translations and no significant reduction of the tibialis anterior EMG in unexpected compared with expected rotations in both the control and cerebellar patient. There was a tendency of EMG responses to be larger in the unexpected perturbations suggesting response "habituation" with repetition. Note the large postural response size and coactivation of the tibialis anterior and gastrocnemius muscle after platform translations in the cerebellar patient.

tional conditions were not significantly different from zero for both the control $(0-25 \mathrm{~ms}$ : $\mathrm{p}=0.36,0-75$ ms: $\mathrm{p}=0.13$ ) and cerebellar group (0-25 ms: $\mathrm{p}=0.11,0-75 \mathrm{~ms}$ : 0.18). There were also no significant differences between groups (0-25 ms: $\mathrm{p}=0.06,0-75 \mathrm{~ms}$ : $\mathrm{p}=0.32$ )

\section{Discussion}

The findings of the present study showed that cerebellar dysfunction did not lead to disturbances of adaptation of postural responses to changes in the type of support surface displacements. Both controls and cerebellar patients showed an immediate change in the pattern of automatic postural responses on the first trial when the surface perturbation changed from translation to rotation and vice versa. Neither the ("functionally appropriate") gastrocnemius nor the tibialis anterior response increased over sequential trials to platform translations following platform rotations and vice versa as might be expected if gradual, functional "tuning" of response magnitude occurred. The amplitude of the ("functionally inappropriate") gastrocnemius muscle (and tibialis anterior) response was immediately reduced on the first trial when the perturbation changed from a translation to a rotation (and vice versa). There was a tendency for the tibialis anterior response to gradually decline over subsequent trials of platform perturbations. Trial effects were less prominent in the gastrocnemius muscle responses and did not reach significance. The "functionally inappropriate" gastrocnemius muscle response did not yield a permanent disappearance of the response to subsequent platform rotations.

The results of protocol 1 ("blocked") were further supported by the findings in protocol 2 ("expected $v$ unexpected"). The "functionally appropriate" gastrocnemius muscle (or tibialis anterior) responses were not significantly smaller in unexpected translations (or rotations) compared with expected translations (or rotations). Thus, neither controls nor cerebellar patients showed effects of prediction based on prior experience in early postural responses to unexpected directions of surface displacements. Most of the functional adaptation to changing types of perturbations occurred immediately.

Our results are in contrast with Nashner's previous findings in both controls and cerebellar patients. ${ }^{23} \mathrm{He}$ demonstrated a progressive increase of the "functional" and elimination of the "non-functional" postural muscle responses within three to five sequential trials in healthy control subjects and impaired functional adaptation of postural responses to changing types of perturbations in a small group of cerebellar patients.

Previous studies have already challenged Nashner's findings of progressive alterations of postural responses to changes in perturbation types over succeeding trials in healthy subjects. Consistent with our findings, these studies showed an immediate change in postural response pattern within the first trial when the type of perturbation unexpectedly changed..$^{4-8}$ Nashner's and Nashner and Grimm's example of sequentially increasing gastrocnemius muscle response to backward translations might be explained by lack of control for the initial body position: Subjects are likely to lean forward if they expect a backward translation based on prior experience. Preleaning stretches the gastrocnemius muscle muscle and results in an increase of the gastrocnemius muscle response. ${ }^{19} 20$

Comparable with our findings, a reduction of both "functionally appropriate" and "inap- 
propriate" postural response amplitudes over succeeding trials has been shown by several other authors. ${ }^{41921}$ Hansen et al demonstrated an initial reduction of the gastrocnemius muscle response between trial 1 and subsequent rotational trials, which could be due to habituation of a startle-like response, and a second more gradual reduction over ongoing trials. Consistent with our findings, neither Diener et al nor Hansen et al found a permanent disappearance of the gastrocnemius muscle response to subsequent platform rotations following translations. ${ }^{4} 19$

Nashner's finding of total disappearance of the gastrocnemius muscle response over sequential rotational perturbations might be explained by the great variability of the gastrocnemius response in particular to the smaller and slower perturbations used by Nashner. ${ }^{21922}$ The response amplitude to a rotational perturbation has been demonstrated to be linearly related to the displacement amplitude and velocity. ${ }^{19}$ The velocity of platform perturbation was significantly faster in our and previous studies compared with Nashner's study for both translations and rotations (present study: rotation $4^{\circ}, 50 / \mathrm{s}$; translation: $2.7 \mathrm{~cm}, 40 \mathrm{~cm} / \mathrm{s}$; Nashner: rotation: $1.8^{\circ}$, 6/s, translation: "at a fixed rate to produce sway at $1 / \mathrm{s}$ during a $500 \mathrm{~ms}$ trial period"). The faster velocity might have prevented total disappearance of the response within five trials. On the other hand, the gastrocnemius muscle response to toes up platform rotations has been shown to be variable and occasionally missing in normal subjects. ${ }^{419} 22$ Nashner used a short sequence of perturbations of only four to five trials and therefore could have missed the finding of the reappearance and the continued variability of the responses.

Nashner's findings in cerebellar patients have often been used to underline the role of the cerebellum in the organisation of muscle synergies for coordinated movements. ${ }^{9}{ }^{10}$ However, we could neither verify gradual adaptation of postural response synergies in normal subjects nor show impairment in changing from one automatic postural response strategy to the other in cerebellar patients. In addition to the arguments for normal subjects discussed above, the different findings in patients with cerebellar dysfunction are likely to be due to different patient populations. Nashner and Grimm included patients with additional extracerebellar lesions, whereas only patients with circumscribed cerebellar lesions were included in the present study.

Changes in types of perturbations result in changes of proprioceptive as well as visual and vestibular afferent information because of the difference in ankle and head displacements during platform rotation or translation. ${ }^{623} 24$ Based on their findings in healthy subjects, several authors concluded that initial compensation to a new perturbation type occurs within the first trial by the integration of the divergent sensory inputs. ${ }^{467}$ Our findings in cerebellar patients suggest that the integrity of the cerebellum might not be critical for the integration of afferent inputs elicited by changes in perturbation directions causing immediate changes in postural synergies. These findings are consistent with previous results showing that encoding of velocity feedback of the current trial to scale the amplitude of early postural responses is not impaired in cerebellar patients despite their hypermetria. ${ }^{12}$

The present results support previous findings of our group suggesting that the main role of the cerebellum in automatic postural responses might be gain control rather than organisation, adaptation or selection of postural synergies. In cerebellar patients, postural responses are known to be hypermetric whereas the temporal relation of hip, knee, and ankle muscles of early automatic postural responses are not affected. ${ }^{12} 1425$ Likewise, in the present study, EMG burst magnitude, duration, and cocontraction were larger in cerebellar patients than normal subjects with no difference in postural response latencies.

We recently reported that cerebellar patients' deficits in scaling the magnitude of their early automatic postural responses to the predicted amplitudes of surface translations based on central set from prior experience related to the importance of the cerebellum in accurately modifying the response gain and not in their ability to use prediction. ${ }^{14}$ Furthermore, the cerebellum was not critically involved in the integration of the descending central command to step with the ascending peripheral information associated with the platform perturbation. ${ }^{25}$

These results are in line with previous findings in cerebellar patients which showed preserved adaptation to misleading visual stimuli $^{26}$ and deep respiration, ${ }^{27}$ despite postural hypermetria. Furthermore, studies using electrical stimulation of the tibial nerve in standing subjects demonstrated an increased gain of long latency reflexes and did not support Nashner's findings of abnormal fixed patterns of rapid postural responses. ${ }^{28} 29$

In the present study, the ability to modify postural synergies immediately with changes in the type of perturbation was preserved in cerebellar patients despite their hypermetria. Therefore, the cerebellum may not be critically involved in adaptation of postural synergies to changing stimulus types. The cerebellum might be mainly involved in the control of the magnitude or gain of early automatic postural responses.

We thank H-G Elles and C Riedel for technical support, Dr H Schicks for her help in conducting the initial experiments, and J Drepper for conducting the statistical analysis. librium. In: Rowell JL, Shepard J, eds. Handbook of physiology: section 12. Exercise: regulation and integration of multiple systems. New York: American Physiological Society, 1996:255-92

2 Nashner LM. Adapting reflexes controlling the human posture. Exp Brain Res 1976;26:59-72.

3 Nashner LM, Grimm RJ. Analysis of multiloop dyscontrol in standing cerebellar patients. In: Desmedt JE, ed. Cerebral motor control in man: long loop mechanism. Progress in Clinical Neurophysiology 1978;4:300-19.

4 Hansen PD, Woollacott M, Debu B. Postural responses to changing task conditions. Exp Brain Res 1988;73:627-36.

5 Gollhofer A, Horstmann GA, Berger W, et al. Compensation of translational and rotational perturbations in human posture: stabilization of the centre of gravity. Neurosci Lett 1989;105:73-8. 
6 Nardone A, Giordano T, Corra T, et al. Responses of leg muscles in humans displaced while standing. Effects of types of cles in humans displaced while standing. Effects of typ

7 Derturbation and of postural set. Brain 1990;113:65-84. movements: interaction between central programs and afferent input. Physiol Rev 1992;72:33-68.

8 Prochazka A. Proprioceptive feedback and movement regulation. In: Rowell JL, Shepard J, eds. Handbook of physiology: section 12. Exercise: regulation and integration of multiple systems. New York: American Physiological Society, 1996:89-127.

9 Kandel ER, Schwartz JH, Jessel TM. Principles of neural science. 3rd edition. Norwalk: Appleton and Lange, 1990.

10 Thach WT, Goodkin HP, Keating JG. The cerebellum and the adaptive coordination of movement. Ann Rev Neurosci 1992;15:403-42.

11 Friedemann HH, Noth J, Diener HC, et al. EMG responses in hand and leg muscles. Cerebellar disorders. $f$ Neurol Neurosurg Psychiatry 1987;50:71-7.

12 Horak FB, Diener HC. Cerebellar control of postural scaling and central set in stance. $\mathcal{F}$ Neurophysiol 1994;72:479-93.

13 Horak FB, Diener HC, Nashner LM. Influence of central set on human postural responses. $\mathcal{F}$ Neurophysiol 1989;62: 841-53.

14 Timmann D, Horak FB. Prediction and set-dependent scaling of early postural responses in cerebellar patients. Brain 1997;120:327-37.

15 Harding AE. Clinical features and classification of inherited ataxias. In: Harding AE, Deufel T, eds. Advances in Neurology. New York: Raven Press, 1993:1-14.

16 Klockgether T, Schroth G, Diener HC, et al. Idiopathic cerebellar ataxia of late onset: natural history and MRI morphology. I Neurol Neurosurg Psychiatry 1990;153:297-305.

17 Timmann D, Belting C, Schwarz M, et al. Influence of visual and somatosensory input on leg EMG responses in

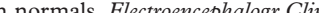
Neurophysiol 1994;93:7-14

18 Timmann D, Plummer C, Schwarz M, et al. Influence of flupirtine on human lower limb reflexes. Electroencephalogr Clin Neurophysiol 1995;97:184-8.
19 Diener HC, Dichgans J, Bootz F, et al. Early stabilization of human posture after a sudden disturbance: influence of rate and amplitude of displacement. Exp Brain Res 1984;56:126-34.

20 Horak FB, Moore SP. The effect of prior leaning on human postural responses. Gait and Posture 1993;1:203-10.

21 Horstmann GA, Gollhofer A, Dietz V. Reproducibility and adaptation of the EMG responses of the lower leg following perturbations of upright stance. Electroencephalogr Clin Neurophysiol 1988;70:447-52.

22 Allum JHJ, Büdingen HJ. Coupled stretch reflexes in ankle muscles: an evaluation of active muscle mechanisms to human posture. In: Granit R, Pompeiano O, eds. Progress in Brain Research. Vol 50. New York: Elsevier, 1979:185-96.

23 Allum JHJ, Pfaltz CR. Visual and vestibular contributions to pitch sway stabilization in the ankle muscles of normals and patients with bilateral peripheral vestibular deficits. Exp Brain Res 1985;58:82-94.

24 Keshner EA, Woollacott MH, Debu B. Neck, trunk and imb muscle responses during postural perturbations in humans. Exp Brain Res 1988;71:455-66.

25 Timmann D, Horak FB. Perturbed step initiation in cerebellar subjects: 1 . Modifications of postural responses. Exp Brain Res 1998;119:73-84.

26 Bronstein AM, Hood JD, Gresty MA, et al. Visual control of balance in cerebellar and Parkinsonian syndromes. Brain 1990;113:767-79.

27 Gurfinkel VS, Elner AM On two types of statics disturbances in patients with local lesions of the brain. Agressologie 1973;14:65-72.

28 Mauritz KH, Dichgans J, Hufschmidt A. Quantitative analysis of stance in late cortical cerebellar atrophy of the anterior lobe and other forms of cerebellar ataxia. Brain 1979;102:461-82.

29 Mauritz KH, Schmitt C, Dichgans J. Delayed and enhanced long latency reflexes as the possible cause of postural tremor in late cerebellar atrophy. Brain 1981;104:97-116. 